\title{
Development of a Traditional Chinese Medicine Syndrome-Specific Scale for Ulcerative Colitis: The Large Intestine Dampness-Heat Syndrome Questionnaire
}

\author{
Xin-lin Chen, ${ }^{1}$ Yi Wen $\mathbb{D}^{2},{ }^{2} \mathrm{Zu}$-chun Wu, ${ }^{1}$ Bei-ping Zhang, \\ Zheng-kun Hou $\mathbb{D}^{4}{ }^{4}$ Jun-lin Xiao, ${ }^{5}$ Man-qing Lin, ${ }^{2}$ Yue Hu, ${ }^{1}$ Zhe-li Wu, ${ }^{1}$ \\ Jie-min Deng, ${ }^{1}$ Feng-bin Liu $\left(\mathbb{D},{ }^{4}\right.$ and Tian-wen Liu $\mathbb{D D}^{3}$ \\ ${ }^{1}$ School of Basic Medical Science, Guangzhou University of Chinese Medicine, Guangzhou, China \\ ${ }^{2}$ The First Clinical College, Guangzhou University of Chinese Medicine, Guangzhou, China \\ ${ }^{3}$ The Second Affiliated Hospital, Guangzhou University of Chinese Medicine, Guangzhou, China \\ ${ }^{4}$ The First Affiliated Hospital, Guangzhou University of Chinese Medicine, Guangzhou, China \\ ${ }^{5}$ The Second Clinical College, Guangzhou University of Chinese Medicine, Guangzhou, China \\ Correspondence should be addressed to Feng-bin Liu; liufb163@163.com and Tian-wen Liu; liutianwen100@163.com
}

Received 23 March 2018; Revised 21 May 2018; Accepted 28 June 2018; Published 12 July 2018

Academic Editor: Raffaele Capasso

Copyright (c) 2018 Xin-lin Chen et al. This is an open access article distributed under the Creative Commons Attribution License, which permits unrestricted use, distribution, and reproduction in any medium, provided the original work is properly cited.

\begin{abstract}
The aim of this study was to develop and validate the large intestine dampness-heat syndrome questionnaire (LIDHSQ) for patients with ulcerative colitis (UC). The domains and items of the LIDHSQ were developed according to standard procedures, namely, construct definition, item generation, language testing, content validity, pilot study, and validation study. At first, a total of 20 items in 3 domains were generated based on literature review and expert consultation. After the item selection, the LIDHSQ contains 11 items in three domains: disease-related domain (diarrhoea, abdominal pain, bloody purulent stool, and mucus stool), heat domain (fever, dry mouth, red tongue, yellow fur, and anal burning), and dampness domain (greasy fur and defecation disorder). The Cronbach's alphas of all domains were greater than 0.6. All of the intraclass correlation coefficients were greater than 0.8. The LIDHSQ and domain scores of the patients with LIDHS were higher than those of the patients with other syndromes $(P<0.001)$. The area under the receiver operating characteristic curve of the LIDHSQ was 0.900 , with a $95 \%$ confidence interval of $0.872-0.928$. When the cut-off value of the LIDHSQ was $\geq 7$, the sensitivity and specificity were 0.867 and 0.854 , respectively. The LIDHSQ is valid and reliable for measuring LIDHS in UC patients with good diagnostic efficacy. We recommend the use of the LIDHSQ in Chinese UC patients.
\end{abstract}

\section{Introduction}

Ulcerative colitis (UC) is an idiopathic, chronic inflammatory disorder of the colonic mucosa that starts in the rectum and generally extends proximally in a continuous manner through either part of or the entire colon. In the West, the incidence and prevalence of UC have increased over the past 50 years, up to $8-14 / 100,000$ and $120-200 / 100,000$ persons, respectively [1]. Some studies have reported that the incidence and prevalence rates of UC have increased gradually in China [2-5]. The incidence of UC varied from
0.07 to 4.90 per 100,000 people per year in China based on the results of a multinational, population-based study conducted between 2011 and 2013 [3]. It was estimated that the prevalence of UC in China is 11.6 per 100,000 people in 2016 [4]. Large intestine dampness-heat syndrome (LIDHS) is one of the most common UC syndromes [6-9]. Chen et al. reported that LIDHS accounted for $34.8 \%$ of the UC patients according to the results of a literature review [6].

Traditional Chinese medicine (TCM) is widely used to treat UC as a complementary and alternative medicine [1016]. Some clinical research has reported that TCM therapies 


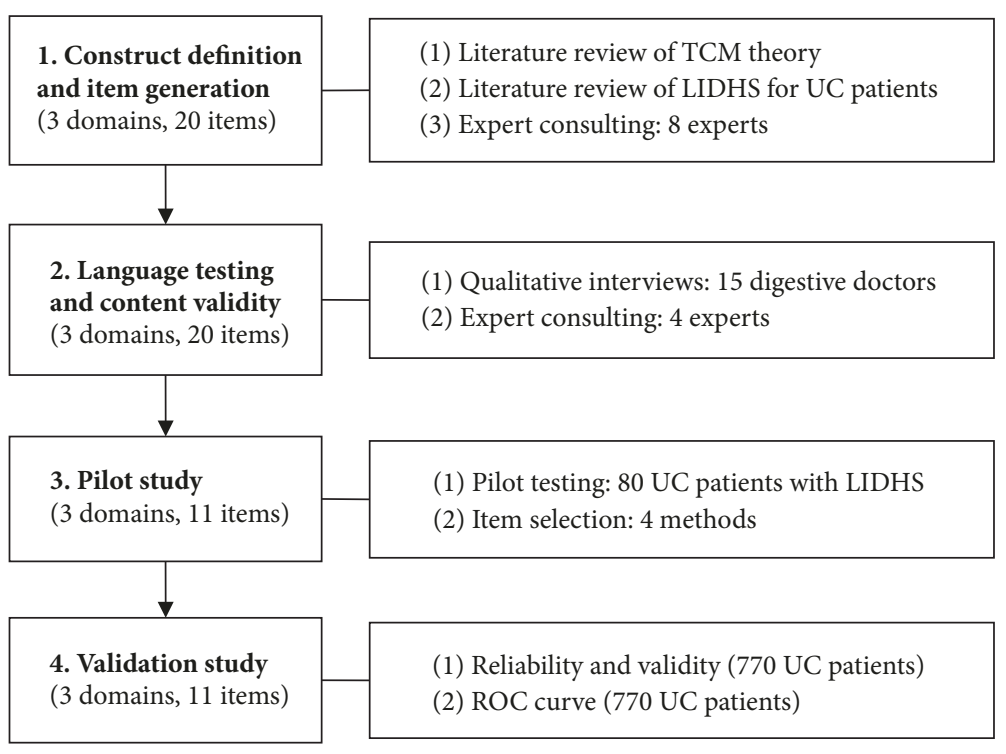

Figure 1: Four steps towards development of the procedure.

are effective for treating UC patients with LIDHS in the clinic $[8,13,14]$. Chinese herbal medicine can effectively inhibit intestinal inflammation for UC patients with LIDHS $[8,14]$.

The mainstay of LIDHS diagnosis was based on the experience of TCM doctors. However, the diagnosis of LIDHS is problematic. First, the diagnostic criteria for LIDHS in UC patients are not uniform. Five diagnostic criteria for LIDHS can be found in the literature [17-21]; however, these criteria all contain different items. For example, mucus stool was the main item in three criteria $[17,18,21]$ but was not found in the others $[19,20]$. The lack of consistent criteria increases the difficulty of diagnosing LIDHS. Second, there is a lack of validated and standardized LIDHS-specific scales in TCM clinical practice.

Some researchers have reported that the standard development and validation procedures for health-related quality of life (HRQOL) or patient-reported outcomes (PRO) questionnaires can be effectively used to develop and assess TCM outcomes and syndromes [22-29]. For example, our team developed and validated TCM outcomes using these procedures $[22,25]$. Diagnostic tests are a type of medical procedure performed for the diagnosis of a disease $[30,31]$ and are also tools used to diagnose TCM syndromes [32]. The aim of this study was to use standard development and validation procedures to develop and assess the large intestine dampness-heat syndrome questionnaire (LIDHSQ) which was administered by the doctors.

\section{Methods}

The standard procedures for questionnaires were followed to develop and validate the LIDHSQ for UC [33-35]. According to the suggestions of the World Health Organization Quality of Life (WHOQOL) instruments group and the US Food and Drug Administration (FDA), the procedures include the following five steps: (1) hypothesizing the conceptual framework; (2) adjusting the conceptual framework and drafting the instrument; (3) confirming the conceptual framework and assessing other measurement properties; (4) collecting, analysing, and interpreting the data; and (5) modifying the instrument [28, 33-35]. For the development of the LIDHSQ, the procedure included construct definition and item generation, language testing and content validity, a pilot study, and a validation study (Figure 1).

2.1. Construct Definition and Item Generation. The LIDHS refers to the syndrome resulting from dampness and heat attacking large intestine, mainly leading to the dysfunction of the large intestine in transportation of food and waste [36]. Dampness is a pathological substance formed by the invasion of external moist pathogen or abnormal movement of body fluid. Heat can be caused by exogenous Yang-heat pathogenic factors, emotional hyperpolarization, or excesses of visceraQi. The interaction between dampness and heat can lead to a series of clinical symptoms for UC patients, especially those in the gastrointestinal tract, such as abdominal pain, diarrhoea, bloody stool, mucus stool, and nausea and vomiting. The UC patients with LIDHS usually have some symptoms of dampness (such as greasy fur, slippery pulse, soft pulse, and mucus stool) and heat (such as fever, dry mouth, halitosis, red tongue, and anal burning).

The research team was composed of three TCM physicians, two TCM diagnostics educators, and two HRQOL researchers. A literature review and expert consultation were used to establish the domains of the LIDHS. (1) A literature review on LIDHS for UC was conducted. Five criteria for LIDHS in UC patients in guidelines or guidebooks were found [17-21]. (2) Expert consultation was performed. Five TCM digestive doctors and three experts in TCM diagnosis with over 10 years of clinical research and teaching experience were included. The research team reviewed the related TCM scales such as the Chinese Quality of Life Instrument (ChQoL) and the suboptimal health status questionnaire (SHS). Based on the TCM theory and the 
TABLE 1: The 18 items from LIDHS diagnostic criterion for UC patients based on literature review.

\begin{tabular}{|c|c|c|c|c|c|}
\hline Items & 1998 criterion [17] & 2002 criterion [18] & 2005 criterion [19] & 2010 criterion $[20]$ & 2011 criterion [21] \\
\hline Diarrhoea & Yes & Yes & Yes & Yes & Yes \\
\hline Abdominal pain & Yes & Yes & Yes & Yes & Yes \\
\hline Bloody purulent stool & Yes & Yes & Yes & Yes & Yes \\
\hline Mucus stool & Yes & No & No & Yes & Yes \\
\hline Tenesmus & Yes & Yes & Yes & Yes & Yes \\
\hline Anal burning & Yes & Yes & Yes & Yes & Yes \\
\hline Fever & No & Yes & Yes & Yes & Yes \\
\hline Scanty deep-yellow urine & Yes & Yes & Yes & Yes & Yes \\
\hline Bitter taste in mouth & Yes & No & No & Yes & Yes \\
\hline Dry mouth & No & No & No & Yes & Yes \\
\hline Halitosis & No & No & No & Yes & No \\
\hline Red tongue & Yes & Yes & Yes & Yes & Yes \\
\hline Greasy fur & Yes & Yes & Yes & Yes & Yes \\
\hline Yellow fur & Yes & Yes & Yes & Yes & Yes \\
\hline Thick fur & No & No & No & No & Yes \\
\hline Rapid pulse & Yes & Yes & Yes & Yes & Yes \\
\hline Slippery pulse & Yes & Yes & Yes & Yes & Yes \\
\hline Soft pulse & Yes & Yes & Yes & No & Yes \\
\hline
\end{tabular}

Yes: the item exists in the criterion. No: the item does not exist in the criterion.

results of literature review and expert consultation, the team proposed three domains of LIDHS for UC patients: the disease-related domain, heat domain, and dampness domain. The disease-related domain was used to indicate the location of the disease, which can be used to distinguish it from other dampness-heat syndromes, such as spleen-stomach dampness-heat syndrome. The heat domain was related to the diagnosis of heat syndrome, and the dampness domain was related to the diagnosis of dampness syndrome.

Based on the literature review on LIDHS for UC patients, eighteen items were included (Table 1). These items contained diarrhoea, abdominal pain, bloody purulent stool, mucus stool, tenesmus, anal burning, fever, scanty deep-yellow urine, bitter taste in mouth, dry mouth, halitosis, red tongue, greasy fur, yellow fur, thick fur, rapid pulse, slippery pulse, and soft pulse. The experts in expert consultation suggested that another two items (anorexia and defecation disorder) were common TCM symptoms for the UC patients with LIDHS and might be helpful to improve the sensitivity and specificity of the LIDHSQ. Thus, the two items were added. Finally, a total of 20 items were generated and classified into three domains: disease-related domain (five items), heat domain (nine items), and dampness domain (six items). The response options for the items were binary: yes (positive) or no (negative).

2.2. Language Testing and Content Validity. All items were tested by 15 TCM digestive doctors. We ensured that the items could be understood by TCM doctors and that the meaning of the item did not cause ambiguity. The content validity of the items was also assessed by these digestive doctors. Problematic items were modified according to their suggestions. Expert consultation (4 experts) was available to evaluate whether the items could represent the most important aspects of the LIDHSQ for UC [37]. Minor revisions and rewording of some items were performed until content validity was achieved. Finally, the first version of the LIDHSQ was established.

2.3. Pilot Study. A pilot study (cross-sectional study) was conducted to select the items. The UC patients with LIDHS were enrolled. The Research Ethics Committee of Guangzhou University of Chinese Medicine provided ethical approval. All of the patients provided informed consent to participate. Eligibility criteria included the following: (1) diagnosed with UC according to the criteria drafted by the inflammatory bowel disease (IBD) group of the Chinese Medical Association Gastroenterology Branch (CMAGB) [38], (2) diagnosed with LIDHS according to the criteria drafted by spleen and stomach disease branch of the China Association of Chinese Medicine (CACM) [20], (3) aged $\geq 12$ years, and (4) providing informed consent to participate. The patients were excluded if they were diagnosed with other syndromes or other diseases.

The items were screened and selected using the following four methods: (1) The proportion of positive responses for each item was calculated, and the items with proportion $<0.4$ were marked. (2) At first, we calculated the internal consistency coefficient ( $\alpha$ value) of all the items. At each time, one of the items was deleted. And then we calculated the new $\alpha$ value and observed the change of $\alpha$ value for each item deleted. The item with the change of $\alpha$ value $>0$ was marked. (3) Correlation coefficient ( $r$ ) between each item and its corresponding domain was calculated, and the item with $\mathrm{r}$ $<0.4$ was marked. (4) Confirmatory factor analysis (CFA) of each item was performed, and the items with loading factor $<0.5$ were marked. When the item meets two or more of the above criteria, it will be finally removed. 
2.4. Validation Study. A validation study was conducted to assess the psychological characteristics of the LIDHSQ. The Research Ethics Committee of Guangzhou University of Chinese Medicine provided ethical approval. The patients were enrolled from the First Affiliated Hospital and the Second Affiliated Hospital of Guangzhou University of Chinese Medicine. The inclusion criteria of the patients were the following: (1) diagnosed with UC according to the criterion of the IBD group of the CMAGB [38] and diagnosed with LIDHS or other syndromes [20]; (2) aged > 12 years; (3) not affected by other infectious diseases; (4) and providing informed consent to participate. The exclusion criteria were the following: (1) diagnosed with other diseases (e.g., Crohn's disease); (2) having another severe disease or complicated polymorbidities with polar outcomes, such as cancer, cardiovascular disease, diabetes, renal disease, or liver disease; and (3) being pregnant or nursing.

The experienced TCM doctors (working for more than 5 years) evaluated the syndromes (LIDHS and other syndromes) of UC patients according to the criteria drafted by the spleen and stomach disease branch of the CACM [20]. To ensure the consistency and accuracy of the TCM syndromes for UC patients, the syndrome of each patient was diagnosed by two experienced doctors. If there was inconsistency, a third chief doctor (associate professor or above) resolved the disagreement. The diagnostic results of the doctors were considered as the gold standard.

All of the patients were also assessed by the TCM doctor using the LIDHSQ. Twenty-two inpatients were assessed using the LIDHSQ within 7-10 days, which was used to assess test-retest reliability. The patients completed a sociodemographic form without assistance. The sociodemographic form covered age, sex, marital status, occupation, smoking, drinking, dietary preferences, and family history.

SPSS for Windows (version 22.0) and Lisrel software (version 8.7) were used for the analysis. The Kappa coefficient was applied to calculate the agreement between the two experts. The percentage of missing data and the frequency of each item were described. The correlation coefficients between the item and its own domain or other domains were calculated. Cronbach's $\alpha$ value was used to evaluate internal consistency reliability. Test-retest reliability was assessed using the intraclass correlation coefficient (ICC) and its $95 \%$ confidence interval (CI). Construct validity was assessed using root mean square error of approximation (RMSEA) and some fit indexes based on structural equation modelling (SEM) [39, 40]. The domain scores between patients with LIDHS and those with other syndromes were compared using $t$-tests. Diagnostic tests were used to test the diagnostic value (sensitivity and specificity). The cut-off value was also calculated.

\section{Results}

3.1. Pilot Study. Eighty UC patients with LIDHS were included in the pilot study (Table 2). The mean age of the patients was 48.3 (range: 18-85) years. Fifty patients were male, and 75 were married.
The results of item selection were shown in Table 3. After item selection, nine items, namely, tenesmus, scanty deep-yellow urine, bitter taste in mouth, halitosis, yellow fur, thick fur, rapid pulse, slippery pulse, and soft pulse, were deleted. The LIDHSQ for UC patients contained 11 items covering three domains: The disease-related domain included diarrhoea (item 1), abdominal pain (item 2), mucus in stool (item 3), and bloody purulent stool (item 4). The heat domain included anal burning (item 5), red tongue (item 6), yellow fur (item 7), fever (item 8), and dry mouth (item 9). The dampness domain included greasy fur (item 10) and defecation disorder (item 11). The scores on each domain (or the LIDHSQ) were summed to yield a score for the domain (or the LIDHSQ). The final version of the LIDHSQ is shown in the Supplementary Materials (Table S1).

3.2. Validation Study. A total of 780 UC patients were included in the validation study. Ten patients refused to participate in the study due to limited time. In all, 770 UC patients were included (391 males and 379 females) (Table 2). The mean age of all of the patients was 46.0 (range: 12-86) years. There were 249 patients with LIDHS and 521 patients with other syndromes. The proportion of sexes between patients with LIDHS and those with other syndromes was significantly different $(P<0.001)$. There were no significant differences in age, marital status, occupation, smoking, drinking, dietary preferences, or family history between the two groups $(P>0.05)$.

None of the items had missing data (Table 4). The item "abdominal pain" scored the highest (0.936), followed by diarrhoea (0.928) and red tongue (0.900). The item "fever" scored the lowest (0.402). All of the Kappa coefficients between the two experts were greater than 0.9 (Table 4). The correlation coefficients of each item with its own domain $(P<0.001)$ were greater than those of each item with other domains.

The Cronbach's $\alpha$ value of the LIDHSQ was 0.68 (Table 5). The $\alpha$ values of all domains were greater than 0.6 and were statistically significant $(P<0.05)$. All of the ICCs were greater than 0.8 and were significantly different $(P<0.05$, Table 5$)$. All domain scores and LIDPSQ scores of the patients with LIDPS were higher than those of the patients with other syndromes. All of the scores between the two groups were significantly different $(P<0.001$, Table 6$)$.

The chi-square value was $123.8(P=0.001)$ based on the SEM results. The RMSEA was equal to 0.090 (90\% CI: 0.072-0.109). The comparative fit index (CFI), normed fit index (NFI), and incremental fit index (IFI) were equal to $0.839,0.829$, and 0.824 , respectively. The minimum factor loading was 0.33 (item 8 , Fever). The factor loadings of the other items were greater than 0.4 . The structure diagram is shown in Figure 2.

The receiver operating characteristic (ROC) curve of the LIDHSQ was 0.900 (standard error $=0.014$ ), with a $95 \%$ CI of $0.872-0.928(P<0.001)$. The ROC curve is shown in Figure 3. 
TABLE 2: Characteristics of the included patients.

\begin{tabular}{|c|c|c|c|c|}
\hline \multicolumn{5}{|c|}{$\begin{array}{ll}\text { Pilot study } \\
\end{array}$} \\
\hline & LIDHS $(\mathrm{n}=80)$ & LIDHS ( $\mathrm{n}=249)$ & Other syndromes $(\mathrm{n}=521)$ & $P$ value \\
\hline Age (years, $\bar{x} \pm s$ ) & $48.3 \pm 16.9$ & $46.4 \pm 15.3$ & $45.8 \pm 16.2$ & 0.655 \\
\hline \multicolumn{5}{|l|}{ Sex } \\
\hline Male & $50(62.5)$ & $152(61.0)$ & $239(45.9)$ & \multirow[t]{2}{*}{$<0.001$} \\
\hline Female & $30(37.5)$ & $97(39.0)$ & $282(54.1)$ & \\
\hline \multicolumn{5}{|l|}{ Marital status } \\
\hline Married & $75(93.8)$ & $223(89.6)$ & $455(87.3)$ & \multirow[t]{2}{*}{0.373} \\
\hline Unmarried & $5(6.3)$ & $26(10.4)$ & $66(12.7)$ & \\
\hline \multicolumn{5}{|l|}{ Occupation } \\
\hline Employed & $28(35.0)$ & $92(36.9)$ & $156(29.9)$ & \multirow[t]{5}{*}{0.254} \\
\hline Retiree & $11(13.8)$ & $33(13.3)$ & $68(13.1)$ & \\
\hline Student & $1(1.3)$ & $7(2.8)$ & $25(4.8)$ & \\
\hline Unemployed & $26(32.5)$ & $70(28.1)$ & $155(29.8)$ & \\
\hline Unclear & $14(17.5)$ & $47(18.9)$ & $117(22.5)$ & \\
\hline \multicolumn{5}{|l|}{ Smoking } \\
\hline Yes & $13(16.3)$ & $32(12.9)$ & $56(10.7)$ & \multirow[t]{2}{*}{0.391} \\
\hline No & $67(83.8)$ & $217(87.1)$ & $465(89.3)$ & \\
\hline \multicolumn{5}{|l|}{ Drinking } \\
\hline Yes & $7(8.8)$ & $24(9.6)$ & $45(8.6)$ & \multirow[t]{2}{*}{0.649} \\
\hline No & $73(91.3)$ & $225(90.4)$ & $476(91.4)$ & \\
\hline \multicolumn{5}{|l|}{ Dietary preference } \\
\hline Yes & $8(10.0)$ & $19(7.6)$ & $60(11.5)$ & \multirow[t]{2}{*}{0.096} \\
\hline No & $72(90.0)$ & $230(92.4)$ & $461(88.5)$ & \\
\hline \multicolumn{5}{|l|}{ Family history } \\
\hline Yes & $2(2.5)$ & $5(2.0)$ & $13(2.5)$ & \multirow[t]{2}{*}{0.676} \\
\hline No & $78(97.5)$ & $244(98.0)$ & $508(97.5)$ & \\
\hline
\end{tabular}

LIDHS: large intestine dampness-heat syndrome.

TABLE 3: The results of the item selection (pilot study).

\begin{tabular}{|c|c|c|c|c|c|}
\hline Items & $\mathrm{p}$ & $\alpha$ value & $\mathrm{r}$ & CFA & Reserved item \\
\hline Diarrhoea & 0.96 & -0.06 & 0.49 & 0.35 & Yes \\
\hline Abdominal pain & 0.96 & -0.02 & 0.67 & 0.40 & Yes \\
\hline Bloody purulent stool & 0.93 & -0.12 & 0.62 & 0.71 & Yes \\
\hline Mucus stool & 0.91 & -0.19 & 0.69 & 0.70 & Yes \\
\hline Tenesmus & 0.71 & 0.12 & 0.41 & 0.25 & No \\
\hline Anal burning & 0.69 & 0.03 & 0.47 & 0.49 & Yes \\
\hline Fever & 0.32 & -0.08 & 0.56 & 0.56 & Yes \\
\hline Scanty deep-yellow urine & 0.09 & -0.02 & 0.32 & 0.09 & No \\
\hline Bitter taste in mouth & 0.33 & -0.16 & 0.67 & 0.41 & No \\
\hline Dry mouth & 0.75 & -0.16 & 0.65 & 0.51 & Yes \\
\hline Halitosis & 0.04 & -0.03 & 0.34 & 0.14 & No \\
\hline Red tongue & 0.94 & -0.03 & 0.45 & 0.73 & Yes \\
\hline Greasy fur & 0.73 & -0.04 & 0.55 & 0.25 & Yes \\
\hline Yellow fur & 0.94 & -0.01 & 0.46 & 0.70 & Yes \\
\hline Thick fur & 0.32 & 0.05 & 0.47 & 0.26 & No \\
\hline Rapid pulse & 0.60 & 0.11 & 0.24 & 0.29 & No \\
\hline Slippery pulse & 0.51 & -0.08 & 0.35 & 0.27 & No \\
\hline Soft pulse & 0.36 & 0.07 & 0.23 & -0.07 & No \\
\hline Defecation disorder & 0.43 & -0.05 & 0.60 & 0.34 & Yes \\
\hline Anorexia & 0.33 & -0.10 & 0.40 & -0.24 & No \\
\hline
\end{tabular}

p: the proportion of positive responses; $\alpha$ value: internal consistency coefficients; $r$ : correlation analysis; CFA: confirmatory factor analysis. When the item meets two or more of the above criteria, it will be finally removed. 
TABLE 4: The scores and correlation coefficients for each item $(n=249)$.

\begin{tabular}{|c|c|c|c|c|c|c|c|c|}
\hline & Score of 0 & Score of 1 & Mean & $\begin{array}{l}\text { Standard } \\
\text { deviation }\end{array}$ & Карра* & $\begin{array}{c}\text { Disease- } \\
\text { related } \\
\text { domain }^{\wedge}\end{array}$ & $\begin{array}{c}\text { Heat } \\
\text { domain }^{\wedge}\end{array}$ & $\begin{array}{c}\text { Dampness } \\
\text { domain }^{\wedge}\end{array}$ \\
\hline Diarrhoea & 18 & 231 & 0.928 & 0.259 & 0.878 & 0.684 & 0.253 & 0.169 \\
\hline Abdominal pain & 16 & 233 & 0.936 & 0.246 & 0.934 & 0.697 & 0.134 & 0.094 \\
\hline Mucus stool & 28 & 221 & 0.888 & 0.317 & 0.926 & 0.751 & 0.153 & 0.090 \\
\hline $\begin{array}{l}\text { Bloody purulent } \\
\text { stool }\end{array}$ & 29 & 220 & 0.884 & 0.321 & 0.904 & 0.749 & 0.117 & 0.121 \\
\hline Anal burning & 73 & 176 & 0.707 & 0.456 & 0.940 & 0.185 & 0.650 & 0.030 \\
\hline Red tongue & 25 & 224 & 0.900 & 0.301 & 0.923 & 0.224 & 0.561 & 0.118 \\
\hline Yellow fur & 28 & 221 & 0.888 & 0.317 & 0.904 & 0.227 & 0.624 & 0.181 \\
\hline Fever & 149 & 100 & 0.402 & 0.491 & 0.945 & 0.075 & 0.620 & 0.364 \\
\hline Dry mouth & 61 & 188 & 0.755 & 0.431 & 0.928 & 0.053 & 0.704 & 0.053 \\
\hline Greasy fur & 76 & 173 & 0.695 & 0.461 & 0.916 & 0.171 & 0.182 & 0.865 \\
\hline $\begin{array}{l}\text { Defecation } \\
\text { disorder }\end{array}$ & 129 & 120 & 0.482 & 0.501 & 0.923 & 0.115 & 0.240 & 0.887 \\
\hline
\end{tabular}

*: Kappa was calculated using the scores assigned by the two experts. ${ }^{\wedge}$ : correlation coefficient between the item and domain scores.

TABLE 5: Cronbach's $\alpha$ coefficient and ICC of each domain.

\begin{tabular}{lccr}
\hline & No. of items & Cronbach's $\alpha(95 \%$ CI $)$ & ICC (95\% CI)* \\
\hline Disease-related domain & 4 & $0.69(0.62-0.75)$ & $0.86(0.83-0.89)$ \\
Heat domain & 5 & $0.61(0.53-0.68)$ & $0.83(0.80-0.86)$ \\
Dampness domain & 2 & $0.70(0.61-0.76)$ & $0.82(0.78-0.86)$ \\
The LIDHSQ & 11 & $0.68(0.62-0.73)$ & $0.88(0.85-0.91)$ \\
\hline
\end{tabular}

ICC: intraclass correlation coefficient. $*$ : results based on 22 inpatients.

TABLE 6: Scores of UC patients with different syndromes.

\begin{tabular}{lccc}
\hline & LIDHS $(n=249)$ & Other syndromes $(n=521)$ & $t$ value $(P$ value $)$ \\
\hline Disease-related domain & $3.63 \pm 0.83$ & $3.15 \pm 0.94$ & $7.03(<0.001)$ \\
Heat domain & $3.65 \pm 1.27$ & $1.32 \pm 1.13$ & $25.69(<0.001)$ \\
Dampness domain & $1.18 \pm 0.84$ & $0.63 \pm 0.58$ & $10.43(<0.001)$ \\
The LIDHSQ & $8.46 \pm 2.06$ & $5.10 \pm 1.61$ & $24.73(<0.001)$ \\
\hline
\end{tabular}

TABLE 7: Results of the diagnostic value test.

\begin{tabular}{lccc}
\hline Gold standard & The LIDHSQ & & \\
& Positive (\%) & Negative (\%) & All \\
\hline Positive & $216(86.7)$ & $33(13.3)$ & 249 \\
Negative & $76(14.6)$ & $445(85.4)$ & 521 \\
\hline
\end{tabular}

Cut-off value of the LIDHSQ $\geq 7$ was diagnosed as positive.

When the cut-off value of the LIDHSQ was $\geq 7$, the sensitivity and specificity were 0.867 and 0.854 , respectively (Table 7 ).

\section{Discussion}

The standard procedures raised by the WHOQOL group and the US FDA have been widely adopted to develop and validate TCM outcomes [22, 24, 25] and TCM diagnostic questionnaires $[23,26]$. The WHOQOL group and the US FDA first proposed to establish the conceptual framework of the questionnaire. Chen et al. advocated using exploratory factor analysis (EFA) to explore the symptom domains of the kidney deficiency syndrome questionnaire [29]. EFA is merely a statistical analysis method in which the domains depend heavily on the included items and the factor extraction method. Differences in items or factor extraction methods will lead to inconsistent results. If relevant variables are omitted in the analysis, or scarcely reliable or redundant items are included, the final number and composition of the domains will be seriously affected [41]. However, the structures of the questionnaires based on the procedures of the WHOQOL group and the US FDA had high reliability 


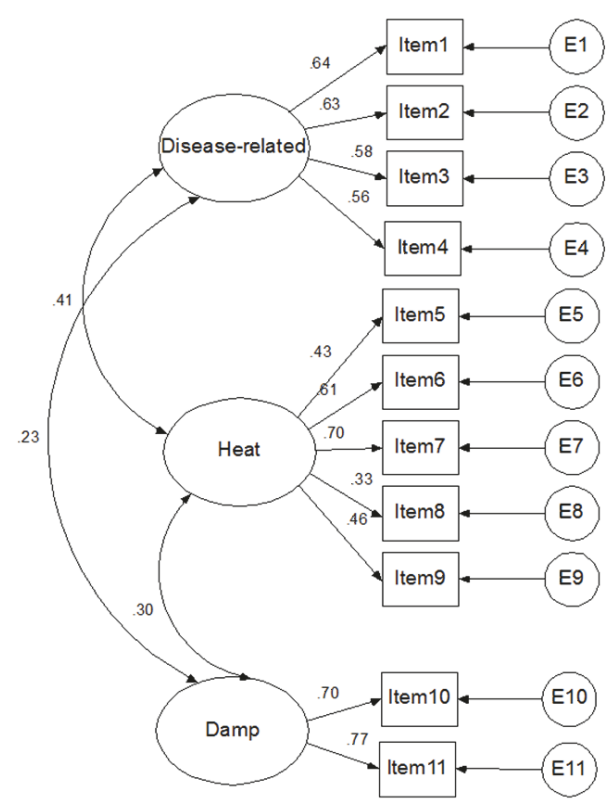

FIGURE 2: Structure diagram (standardized coefficient) of large intestine dampness-heat syndrome (items 1-11: diarrhoea, abdominal pain, mucus stool, bloody purulent stool, anal burning, red tongue, yellow fur, fever, dry mouth, greasy fur, and defecation disorder).

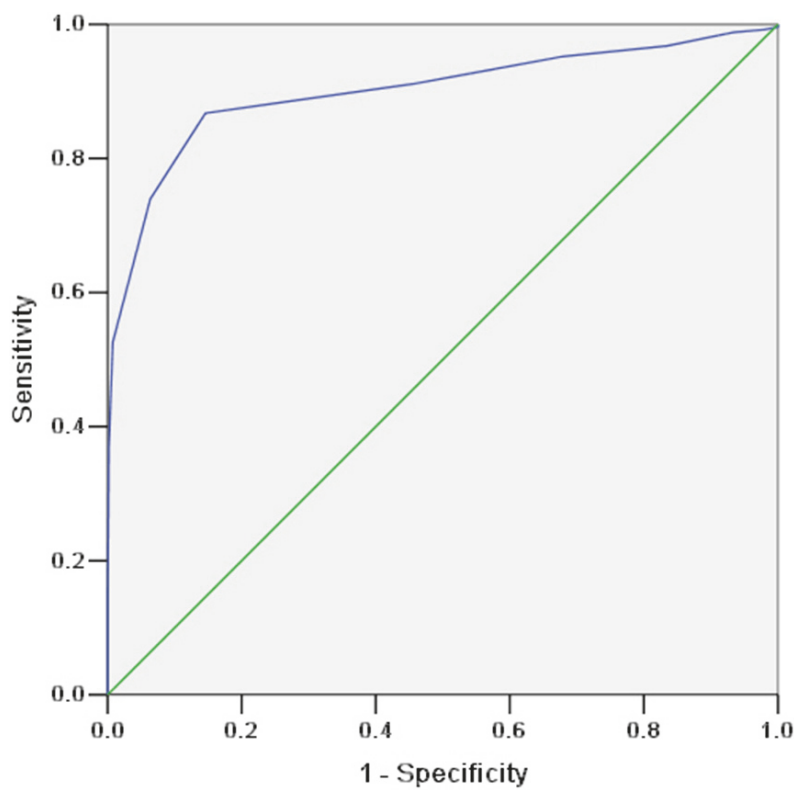

Figure 3: The ROC curve of large intestine dampness-heat syndrome.

and repeatability. Therefore, the procedures of the WHOQOL group and the US FDA were eventually utilized to develop the LIDHSQ.

The LIDHSQ had good content validity according to the review and suggestions of the experts. The LIDHSQ included the most important aspects that characterize LIDHS for the UC patients. The LIDHSQ contained 11 items. (1) The disease-related domain included diarrhoea, abdominal pain, bloody purulent stool, and mucus in the stool, which are the most common bowel symptoms in UC patients. Because UC patients with other syndromes may have had similar symptoms, these items were related to ulcerative colitis. (2) Among these items, fever, dry mouth, red tongue, yellow fur, and anal burning were used to assess heat syndrome. The first four symptoms (fever, dry mouth, red tongue, and yellow fur) are important items for diagnosing heat syndrome according to the theory of TCM. Anal burning could help to locate the intestine. (3) Greasy fur, diarrhoea, and defecation disorder were valuable for diagnosing dampness-heat syndrome in UC patients.

The LIDHSQ had good reliability and validity according to the results. The Kappa coefficients are categorized as very good (0.81 to 1.00$)$, good (0.61 to 0.80$)$, moderate $(0.41$ to 0.60$)$, fair $(0.21$ to 0.40$)$, and poor $(<0.20)$. When the Cronbach's alphas are between 0.70 and 0.95 , the internal consistency is considered good [42]. When ICC is at least 0.70 in a sample size of at least 50 patients, the test-retest reliability is considered to be good [42]. All items had high Kappa coefficients $(>0.9)$ in our results, which indicated that the items had excellent consistency between the TCM doctors. The Cronbach's $\alpha$ of the LIDHSQ was 0.68 , and those of all of the domains were greater than 0.6 . The results showed that the LIDHSQ had acceptable internal consistency. The test-retest reliability coefficients were greater than 0.8 . The RMSEA was equal to 0.090 (90\% CI: 0.072-0.109), which showed that the LIDHSQ had good construct validity. The item fever (item 8) was common in UC patients with LIDHS. Although item 8 (fever) had low loadings (0.33), it was still kept in the LIDHSQ.

The LIDHSQ had good diagnostic efficacy. The cutoff value of the LIDHSQ was $\geq 7$ according to the results of the ROC. Its sensitivity and specificity were 0.867 and 0.854 , respectively. The LIDHSQ was sufficiently sensitive to discriminate the scores of UC patients with different syndromes. The scores of the LIDHSQ (including domains) were higher in the patients with LIDHS than those with other syndromes. These results were consistent with our hypothesis.

The aim of our study was to develop and validate the diagnostic criteria of the LIDHSQ, a questionnaire used in TCM practice. The questionnaire cannot be used to diagnose UC. Therefore, the patients enrolled in our study must first be diagnosed as having UC. It is feasible to establish a theoretical model of TCM syndromes based on the basic theory of TCM, literature review, and expert opinions. TCM syndromes can be treated as latent variables. Using a qualitative item bank, literature review, and expert consultation, we successfully developed the domains and items of the LIDHSQ. The structural equation modelling could be used to quantitatively test TCM syndromes, consistent with other studies [23, 29, 43]. Other researchers have also successfully used similar methods to study TCM syndromes. For example, suboptimal health status (SHS) was characterized by ambiguous health complaints, general weakness, and a lack of vitality. Yan YX et al. developed the SHS questionnaire [24, 44] and indicated that it was a reliable and valid questionnaire as a 
new instrument for predictive, preventive, and personalized medicine (PPPM) of TCM $[45,46]$. The LIDHSQ, as a valid and reliable TCM syndrome scale, may also be applied to PPPM through patient diagnosis and therapy assessment.

There were some limitations in this study. (1) All of the patients in the study were enrolled from the First Affiliated Hospital and the Second Affiliated Hospital of Guangzhou University of Chinese Medicine. Most of the UC patients were from Guangdong Province. The LIDHSQ should be further evaluated using data from other geographical areas in China. (2) Only 249 patients with LIDHS were enrolled in this study. More patients with LIDHS should be enrolled to assess the validity, reliability, and diagnostic efficacy of the questionnaire. Only twenty-two inpatients were included for test-retest reliability. Terwee et al. suggested that $\geq 50$ patients was adequate for the assessment of test-retest reliability [42]. Therefore, more patients with LIDHS should be enrolled to assess test-retest reliability. (3) The response options for the items were binary (positive and negative). The binary data could not describe the symptoms in detail. Multiple choice items should be considered for the future study of the LIDHSQ. (4) No objective (pathological) measures were provided for the diagnosis of LIDHS.

\section{Conclusions}

The LIDHSQ is simple and easy for TCM doctors to perform. The LIDHSQ is valid and reliable for measuring LIDHS in UC patients with good diagnostic efficacy. We recommend the use of the LIDHSQ in Chinese UC patients. Through establishing the LIDHSQ for UC and determining its cut-off value for diagnosis, we hope to diagnose TCM syndromes of $\mathrm{UC}$ in a standardized manner.

\section{Data Availability}

The data used to support the findings of this study are available from the corresponding author upon request.

\section{Disclosure}

Xin-lin Chen and Yi Wen had full access to all of the data in the study and take responsibility for the integrity of the data and the accuracy of the data analysis.

\section{Conflicts of Interest}

The authors declare that there are no conflicts of interest regarding the publication of this paper.

\section{Authors' Contributions}

Xin-lin Chen and Yi Wen were involved in construct definition, validation study, data analysis, and writing of the manuscript. Zu-chun Wu and Bei-ping Zhang were involved in language testing and content validation. Zheng-kun Hou collected the data and modified the manuscript. Jun-lin Xiao, Man-qing Lin, and Yue $\mathrm{Hu}$ collected the data. Zhe-li Wu and Jie-min Deng were involved in pilot testing and item generation. Feng-bin Liu and Tian-wen Liu designed the study. Xin-lin Chen and Yi Wen are equal contributors.

\section{Acknowledgments}

This study was funded by the National Natural Science Foundation of China (81403296 and 81774451), the Outstanding Youth Foundation of Guangdong Province Colleges and Universities (YQ2015041), the Young Talents Foundation of Guangzhou University of Chinese Medicine (QNYC20140101), the Natural Science Foundation of Guangdong Province (2017A030313827), and the Guangdong High Level Universities Program of Guangzhou University of Chinese Medicine. The authors thank Zong-chan Xiu, Lijian Yu, Ya-mei Liu, Kun-hai Zhuang, and Xin Jin from Guangzhou University of Chinese Medicine for their help in improving the items. The authors also thank Cong Sun from Sun Yat-sen University for his help in improving the English language.

\section{Supplementary Materials}

Table S1: the items of the large intestine damp-heat syndrome questionnaire. (Supplementary Materials)

\section{References}

[1] J. Cosnes, C. Gowerrousseau, P. Seksik, and A. Cortot, "Epidemiology and natural history of inflammatory bowel diseases," Gastroenterology, vol. 140, no. 6, pp. 1785-1794, 2011.

[2] K. L. Goh and S.-D. Xiao, "Inflammatory bowel disease: a survey of the epidemiology in Asia," Journal of Digestive Diseases, vol. 10, no. 1, pp. 1-6, 2009.

[3] S. C. Ng, W. Tang, J. Y. Ching et al., "Incidence and phenotype of inflammatory bowel disease based on results from the AsiaPacific Crohn's and colitis epidemiology study," Gastroenterology, vol. 145, no. 1, pp. 158-165.e2, 2013.

[4] G. G. Kaplan and S. C. Ng, "Globalisation of inflammatory bowel disease: perspectives from the evolution of inflammatory bowel disease in the UK and China," The Lancet Gastroenterology \& Hepatology, vol. 1, no. 4, pp. 307-316, 2016.

[5] Q. Ouyang and L. Y. Xue, "Inflammatory bowel disease in the 21 st century in China: turning challenges into opportunities," Journal of Digestive Diseases, vol. 13, no. 4, pp. 195-199, 2012.

[6] X. Chen, C. Zhang, D. Wang et al., "Literature study on the distribution for TCM syndrome of ulcerative colitis," Chinese Archives of Traditional Chinese Medicine, vol. 35, no. 2, pp. 378381, 2017.

[7] J. Wu, X.-Y. Wang, and H.-Y. Sun, "Study on the correlation between Chinese medicine syndrome and the intestinal mucosal manifestations of 137 patients with active ulcerative colitis," Zhongguo Zhong xi yi jie he za zhi Zhongguo Zhongxiyi jiehe zazhi, vol. 32, no. 4, pp. 445-449, 2012.

[8] Z.-Q. Tong, B. Yang, B.-Y. Chen, and M.-L. Zhao, "A multicenter, randomized, single-blind, controlled clinical study on the efficacy of composite sophora colon-soluble capsules in treating ulcerative colitis," Chinese Journal of Integrative Medicine, vol. 16, no. 6, pp. 486-492, 2010.

[9] Z.-H. Yang, P. Yin, and A.-S. Huang, "The Chinese medicine syndrome features of ulcerative colitis by using fluorescence intensity of auto fluorescence imaging," Zhongguo Zhong Xi Yi 
Jie He Za Zhi Zhongguo Zhongxiyi Jiehe Zazhi, vol. 32, no. 10, pp. 1319-1321, 2012.

[10] Y. Gong, Q. Zha, L. Li et al., "Efficacy and safety of Fufangkushen colon-coated capsule in the treatment of ulcerative colitis compared with mesalazine: a double-blinded and randomized study," Journal of Ethnopharmacology, vol. 141, no. 2, pp. 592598, 2012.

[11] Y.-C. Dai, Y.-L. Zhang, L.-J. Wang et al., "Clinical presentation and treatment strategies for ulcerative colitis: a retrospective study of 247 inpatients," Chinese Journal of Integrative Medicine, vol. 22, no. 11, pp. 811-816, 2016.

[12] A. Lindberg, B. Fossum, P. Karlen, and L. Oxelmark, "Experiences of complementary and alternative medicine in patients with inflammatory bowel disease-a qualitative study," $B M C$ Complementary and Alternative Medicine, vol. 14, no. 1, Article ID 407, 2014.

[13] K. Chen and C. Li, "Acupuncture combined with medication for ulcerative colitis with damp-heat syndrome at active phase," Chinese Acupuncture \& Moxibustion, vol. 35, no. 5, pp. 435-438, 2015.

[14] H.-H. He, H. Shen, and K. Zheng, "Observation of the curative effect of qingchang huashi recipe for treating active ulcerative colitis of inner-accumulation of damp-heat syndrome," Zhongguo Zhong Xi Yi Jie He Za Zhi Zhongguo Zhongxiyi Jiehe Zazhi, vol. 32, no. 12, pp. 1598-1601, 2012.

[15] B. Liu, X. Piao, L. Guo et al., "A new chinese medicine intestine formula greatly improves the effect of aminosalicylate on ulcerative colitis," Evidence-Based Complementary and Alternative Medicine, vol. 2017, 8 pages, 2017.

[16] X. Xu, C. Xu, S. M. Saud et al., "Effect of kuijie granule on the expression of TGF- $\beta /$ smads signaling pathway in patients with ulcerative colitis," Evidence-Based Complementary and Alternative Medicine, vol. 2016, 9 pages, 2016.

[17] Chinese Anorectal Association of traditional Chinese medicine, "Diagnosis and treatment of chronic nonspecific ulcerative colitis (draft)," China Journal of Traditional Chinese Medicine (Chinese), vol. 3, no. 4, p. 71, 1998.

[18] X. Y. Zheng, Guiding Principle of Clinical Research on New Drugs of Traditional Chinese Medicine (Chinese), China Press of Medical Sciences and Technology, Beijing, China, 2002.

[19] Digestive Diseases Specialized Committee in Chinese Interactive Medicine Association, "Diagnosis and treatment of ulcerative colitis in Interactive Medicine (Draft)," Chinese Journal of Integrated Traditional \& Western Medicine on Digestion, vol. 13, no. 2, pp. 133-135, 2005.

[20] Spleen and stomach disease branch of China Association of Chinese Medicine, "Consensus on Traditional Chinese Medicine diagnosis and treatment of ulcerative colitis," Journal of Traditional Chinese Medicine and Pharmacy (Chinese), vol. 25, no. 6, pp. 891-895, 2010.

[21] Digestive Diseases Specialized Committee in Chinese Interactive Medicine Association, "The diagnosis and treatment of ulcerative colitis about integrated traditional Chinese and Western Medicine," Chinese Journal of Integrated Traditional \& Western Medicine on Digestion, vol. 19, no. 1, pp. 61-65, 2011.

[22] K.-F. Leung, F.-B. Liu, L. Zhao, J.-Q. Fang, K. Chan, and L.-Z. Lin, "Development and validation of the Chinese Quality of Life Instrument," Health and Quality of Life Outcomes, vol. 3, article no. 26, 2005.

[23] N. L. Zhang, S. Yuan, T. Chen, and Y. Wang, "Latent tree models and diagnosis in traditional Chinese medicine," Artificial Intelligence in Medicine, vol. 42, no. 3, pp. 229-245, 2008.
[24] Y.-X. Yan, Y.-Q. Liu, M. Li et al., "Development and evaluation of a questionnaire for measuring suboptimal health status in urban Chinese," Journal of Epidemiology, vol. 19, no. 6, pp. 333341, 2009.

[25] F.-B. Liu, X.-L. Chen, L. Guo, and X.-B. Liu, "Evaluation of a scale of patient-reported outcomes for the assessment of myasthenia gravis patients in China," Chinese Journal of Integrative Medicine, vol. 18, no. 10, pp. 737-745, 2012.

[26] T. Tomura, K. Yoshimasu, J. Fukumoto et al., "Validity of a diagnostic scale for acupuncture: application of the item response theory to the five viscera score," Evidence-Based Complementary and Alternative Medicine, vol. 2013, 11 pages, 2013.

[27] Q. Guo and Q. Chen, "Standardization of syndrome differentiation defined by traditional chinese medicine in operative breast cancer: a modified delphi study," The Scientific World Journal, vol. 2015, Article ID 820436, 5 pages, 2015.

[28] Y. Su, C.-W. Mo, W.-Q. Cheng et al., "Development and validation of quality of life scale of nasopharyngeal carcinoma patients: the QOL-NPC (version 2)," Health and Quality of Life Outcomes, vol. 14, no. 1, Article ID 76, 2016.

[29] R. Q. Chen, C. M. Wong, and T. H. Lam, "Construction of a traditional Chinese medicine syndrome-specific outcome measure: the Kidney Deficiency Syndrome questionnaire (KDSQ)," BMC Complementary and Alternative Medicine, vol. 12, article 73, 2012.

[30] Y. Wang, J. Xu, R. Guo et al., "Therapeutic effect in patients with coronary heart disease based on information analysis from Traditional Chinese Medicine four diagnostic methods," Journal of Traditional Chinese Medicine, vol. 34, no. 1, pp. 34-41, 2014.

[31] Z. Z. Deng, Y. T. He, and Y. M. Yu, "Comparison between two diagnostic methods of computer's mathematic model and clinical diagnosis on TCM syndromes of rheumatoid arthritis Chinese," Zhongguo Zhong Xi Yi Jie He Za Zhi, vol. 16, no. 12, pp. 727-729, 1996.

[32] X. Liu, D. Peng, Y. Wang et al., "Diagnostic accuracy of chinese medicine diagnosis scale of phlegm and blood stasis syndrome in coronary heart disease: a study protocol," Chinese Journal of Integrative Medicine, pp. 1-6, 2018.

[33] M. Power and W. Kuyken, "World Health Organization Quality of Life Assessment (WHOQOL): development and general psychometric properties," Social Science \& Medicine, vol. 46, no. 12, pp. 1569-1585, 1998.

[34] U.S. Department of Health and Human Services FDA Center for Drug Evaluation and Research, "Guidance for industry: patient-reported outcome measures: use in medical product development to support labeling claims: draft guidance," Health Qual Life Outcomes, vol. 4, p. 79, 2006.

[35] M. J. Power and A. M. Green, "Development of the WHOQOL disabilities module," Quality of Life Research, vol. 19, no. 4, pp. 571-584, 2010.

[36] J. Chen, Diagnosis of Traditional Chinese Medicine, China Press of Traditional Chinese Medicine, Beijing, China, 2015.

[37] S. Magasi, G. Ryan, D. Revicki et al., "Content validity of patientreported outcome measures: perspectives from a PROMIS meeting," Quality of Life Research, vol. 21, no. 5, pp. 739-746, 2012.

[38] Inflammatory bowel disease group of Chinese Medical Association Gastroenterology Branch, "Consensus on the diagnosis and treatment of inflammatory bowel disease (2012, Guangzhou)," Chinese Journal of Gastroenterology (Chinese), vol. 17, no. 12, pp. 763-781, 2012. 
[39] K. G. Jöreskog and A. S. Goldberger, "Estimation of a model with multiple indicators and multiple causes of a single latent variable," Journal of the American Statistical Association, vol. 70, no. 351, part 1, pp. 631-639, 1975.

[40] L. M. Lix, B. Acan Osman, J. D. Adachi et al., "Measurement equivalence of the SF-36 in the canadian multicentre osteoporosis study," Health and Quality of Life Outcomes, vol. 10, article no. 29, 2012.

[41] I. Izquierdo, J. Olea, and F. J. Abad, "Exploratory factor analysis in validation studies: uses and recommendations," Psicothema, vol. 26, no. 3, pp. 395-400, 2014.

[42] C. B. Terwee, S. D. M. Bot, M. R. de Boer et al., "Quality criteria were proposed for measurement properties of health status questionnaires," Journal of Clinical Epidemiology, vol. 60, no. 1, pp. 34-42, 2007.

[43] H. Zhao, W.-H. Xiong, X. Zhao, L.-M. Wang, and J.-X. Chen, "Development and evaluation of a Traditional Chinese Medicine syndrome questionnaire for measuring sub-optimal health status in China," Journal of Traditional Chinese Medicine, vol. 32, no. 2, pp. 129-136, 2012.

[44] V. Kupaev, O. Borisov, E. Marutina, Y.-X. Yan, and W. Wang, "Integration of suboptimal health status and endothelial dysfunction as a new aspect for risk evaluation of cardiovascular disease," EPMA Journal, vol. 7, no. 1, 2016.

[45] Y. Yan, J. Dong, Y. Liu et al., "Association of suboptimal health status with psychosocial stress, plasma cortisol and mRNA expression of glucocorticoid receptor $\alpha / \beta$ in lymphocyte," Stress, vol. 18, no. 1, pp. 29-34, 2014.

[46] O. Golubnitschaja, B. Baban, G. Boniolo et al., "Medicine in the early twenty-first century: Paradigm and anticipation-EPMA position paper 2016," EPMA Journal, vol. 7, no. 1, 2016. 


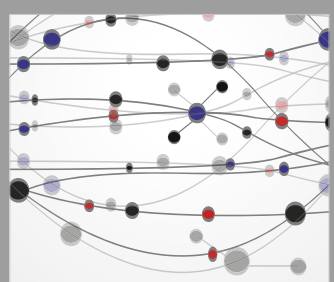

The Scientific World Journal
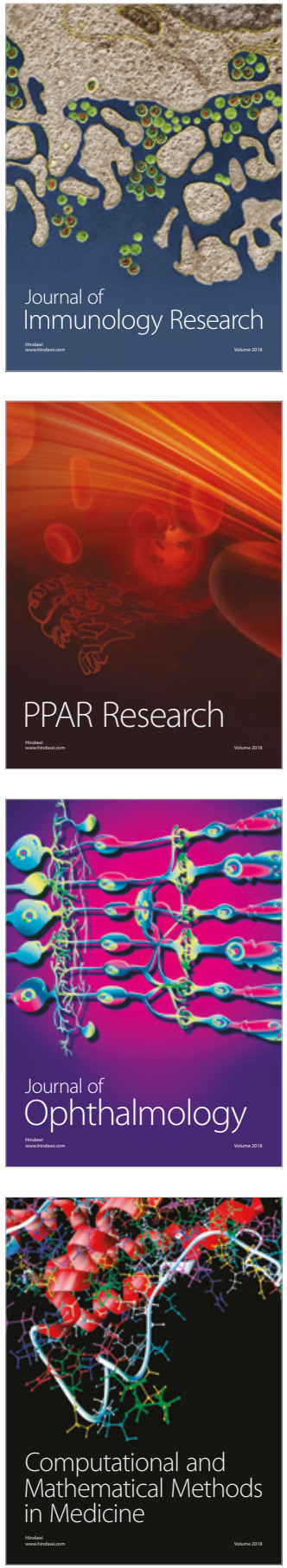

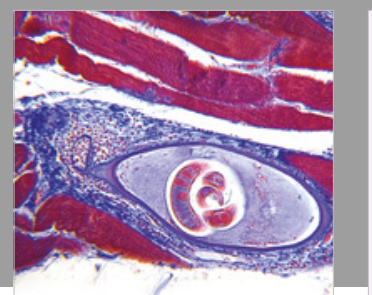

Gastroenterology Research and Practice

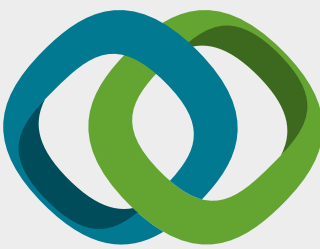

\section{Hindawi}

Submit your manuscripts at

www.hindawi.com
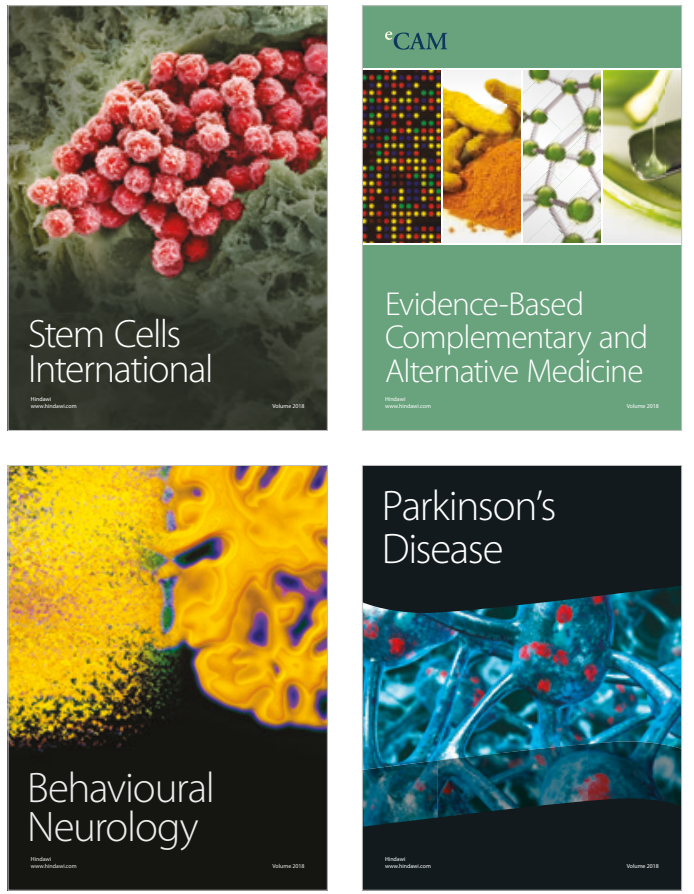

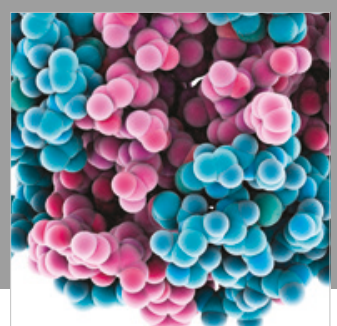

ournal of

Diabetes Research

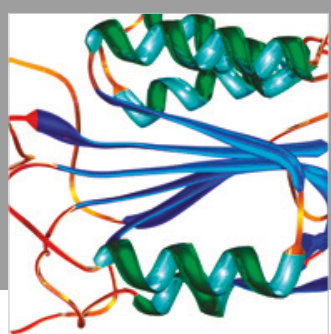

Disease Markers
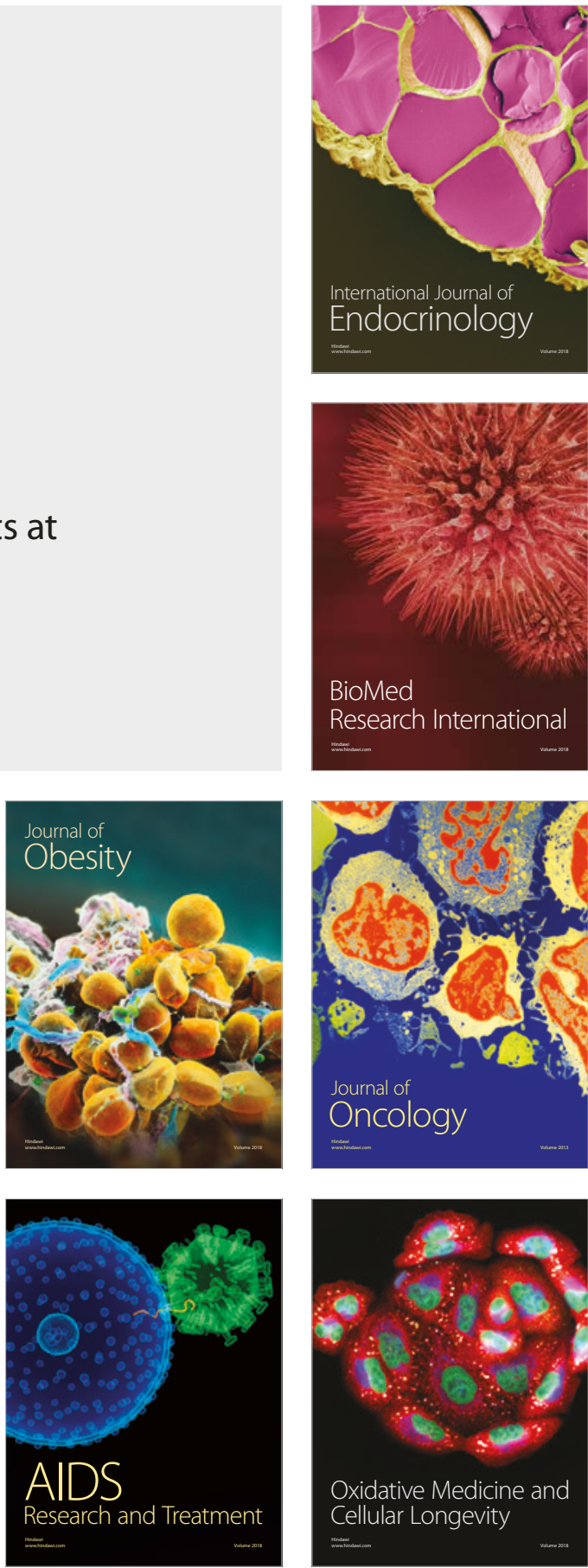